\title{
Waveguide CARS: A Method to Determine the Third-Order Polarizability of Thin Layers Applied to a $\mathrm{SiO}_{x} \mathrm{~N}_{y}$ Waveguide
}

\author{
JOHANNES S. KANGER, CEES OTTO, and JAN GREVE* \\ Applied Optics Group, Department of Applied Physics, BMTI (Biomedical Institute for Technology), MESA (Micro Electronics, \\ Materials Engineering, Sensors \& Actuators), University of Twente, P.O. Box 217, 7500 AE Enschede, The Netherlands
}

\begin{abstract}
Waveguide coherent anti-Stokes Raman spectroscopy (WCARS) can be used to measure Raman-active vibrations of thin (mono, submono) layers deposited on dielectric waveguides. In these experiments, surface sensitivity and background-free spectra are obtained by using asymmetric mode combinations. The degree of suppression then depends upon the waveguide parameters (thicknesses, refractive indices, and third-order polarizabilities), the wavelengths used, and the selected propagating modes. With knowledge of the refractive indices and the third-order polarizabilities, the other parameters can be used to tune into a background-free region. The refractive indices of many waveguide materials are known or can easily be measured. Unfortunately, the third-order polarizabilities are most often not known and should therefore be determined experimentally. In this paper a method is outlined to measure the third-order susceptibility ( $\chi \mathrm{NR}$ ) of a waveguide with the use of WCARS experiments. The value $\chi_{\mathrm{N}}^{3}$ of $\mathrm{SiO}_{x} \mathrm{~N}_{y}$ is determined experimentally and is found to be $0.22 \pm 0.0310^{-12}$ electrostatic units.

Index Headings: Waveguides; Raman; CARS.
\end{abstract}

\section{INTRODUCTION}

Waveguide coherent anti-Stokes Raman spectroscopy (WCARS) is a useful tool to study structure and orientation of thin molecular layers. ${ }^{1}$ The large electrical fields present in dielectric waveguides can induce strong nonlinear effects and therefore can give rise to intense CARS signals. In this way CARS spectra of waveguides made of polymers, with and without additives, have been measured. ${ }^{2,3}$ Another approach is to use the evanescent fields, extending directly above the waveguide surface, to probe Raman-active vibrations of very thin (mono-) layers which are deposited on top of the waveguide. In general, the CARS signal has resonant as well as nonresonant contributions. The resonant part of the signal contains the vibrational information, whereas the nonresonant part yields a constant background. The main drawback of WCARS is the presence of a large nonresonant background originating from the waveguide structure. This nonresonant background signal may obscure the usually weak resonant CARS signals from a monolayer present on top of the waveguide. Because of the discrete polarization possibilities of the guiding modes, background suppression techniques like polarization CARS are not applicable. Destructive interference between different guiding modes may, however, lead to suppression of the signal originating from the waveguide itself., ${ }^{2,4}$ This technique has been applied successfully to measure monolayers on a $\mathrm{ZnO}$ waveguide..$^{5,6}$ Since the quality of the background

\footnotetext{
Received 15 December 1994; accepted 19 May 1995.

* Author to whom correspondence should be sent.
}

suppression depends strongly on the waveguide configuration (refractive indices, layer thicknesses, $\chi_{\mathrm{Nk}}^{(3)}$ ), these parameters must be well defined and determined. A waveguide material for which refractive index and layer thickness can be chosen over a wide range is $\mathrm{SiO}_{x} \mathrm{~N}_{y}$. Therefore, waveguides based on $\mathrm{SiO}_{x} \mathrm{~N}_{y}$ might be a good candidate for waveguide CARS experiments. $\chi_{\mathrm{NR}}^{(3)}$ is not known for this material. This report therefore focuses on a method to determine this property. The method used is based upon the comparison of the unknown $\chi_{\mathrm{Nk}}^{(3)}$ of $\mathrm{SiO}_{x} \mathrm{~N}_{y}$ with the known value of the $\chi^{(3)}$ of polystyrene. To this end, thin layers $(80-500 \mathrm{~nm})$ of polystyrene were spin-coated on top of a $\mathrm{SiO}_{x} \mathrm{~N}_{y}$ waveguide. A comparison of the experimentally observed heterodyned signal with that of the calculated one allows the determination of $\chi_{\mathrm{Nk}}^{(3)}$. In this paper we present the results of these measurements.

\section{THEORY}

In Fig. 1 a three-layered waveguide structure, consisting of a cladding, film, and substrate, is shown together with the electrical field distributions of the three lowest-order guided transverse, or y-polarized, electrical field (TE) modes. The electrical field of a guided TE mode can be written as:

$$
\vec{E}_{\mathrm{p}}^{k}(\mathrm{z}, \mathrm{x}, \omega)=\frac{1}{2} a^{k}(\mathrm{x}) \vec{E}_{0, \mathrm{p}}^{k}(\mathrm{z}) e^{i\left(\omega_{\mathrm{p}} t-\beta_{\mathrm{p}}^{k} \mathrm{x}\right)}+\text { c.c. }
$$

with

$$
\vec{E}_{0, \mathrm{p}}^{k}(\mathrm{z})=\hat{e}_{\mathrm{y}} f_{\mathrm{p}}^{k}(\mathrm{z})
$$

with $\mathrm{p}$ denoting the pump field. An " $\mathrm{s}$ " or " $\mathrm{c}$ " is used for Stokes or CARS fields, respectively. $f^{k}(\mathrm{z})$ is the electrical field distribution function for mode $k ; a^{k}(x)$ is the amplitude distribution function; $\beta^{k}$ is the mode propagation vector of mode $k ; \omega$ is the frequency of the propagating wave; and $k$ enumerates the discrete modes $(k=0,1,2$, ...).

The electrical field distribution function $f^{k}(\mathrm{z})$ is normalized so that $\left|a^{k}(\mathrm{x})\right|^{2}$ gives the guided power of the mode per meter wavefront (in the y direction). In a degenerate WCARS experiment, the pump (p) and Stokes (s) beams are coupled into the waveguide in specific modes (pump: mode $k$; Stokes: mode $l$ ). Where the two beams overlap, a nonlinear polarization is induced at frequency $\omega_{\mathrm{c}}=2 \omega_{\mathrm{p}}-\omega_{\mathrm{s}}$ :

$$
\begin{aligned}
\vec{P}^{(3)}\left(\mathrm{z}, \mathrm{x}, \omega_{\mathrm{c}}\right)= & \overline{\bar{\chi}}^{(3)}\left(z, \omega_{\mathrm{c}}: \omega_{\mathrm{p}}, \omega_{\mathrm{p}},-\omega_{\mathrm{s}}\right): \\
& \vec{E}_{\mathrm{p}}^{k}\left(\mathrm{z}, \mathrm{x}, \omega_{\mathrm{p}}\right) \vec{E}_{\mathrm{p}}^{k}\left(\mathrm{z}, \mathrm{x}, \omega_{\mathrm{p}}\right) \vec{E}_{\mathrm{s}}^{l^{*}}\left(\mathrm{z}, \mathrm{x}, \omega_{\mathrm{s}}\right) .
\end{aligned}
$$


In the following discussion, all frequency notations will be dropped for convenience. The CARS signal (at frequency $\omega_{\mathrm{c}}=2 \omega_{\mathrm{p}}-\omega_{\mathrm{s}}$ ) generated by this polarization is distributed over all possible guiding and radiating modes. With coupled mode theory it can be shown that, for the power, $P_{c}^{m}$ of the CARS signal radiated in the $m$ th guiding mode is given by: ${ }^{7}$

$$
P_{\mathrm{c}}^{m}=\frac{\omega_{\mathrm{c}}^{2}}{16}\left[\frac{L}{H}\right]^{2}\left(P_{\mathrm{p}}^{k}\right)^{2}\left(P_{\mathrm{s}}^{\prime}\right)\left|F^{k l m}\right|^{2} \operatorname{sinc}^{2}\left(\frac{\Delta \beta L}{2}\right)
$$

with $\Delta \beta=2 \beta_{\mathrm{p}}^{k}-\beta_{\mathrm{s}}^{l}-\beta_{\mathrm{c}}^{m}$ denoting the phase mismatch; $L$, the interaction length; $H$, the beam widths; $P_{\mathrm{p}}^{k}$, the power in pump mode $k$; and $P_{\mathrm{s}}^{l}$, the power in Stokes mode $l$; where $F^{k l m}$ is the so-called overlap function and is given by

$$
F^{k l m}=\int_{-\infty}^{+\infty} \overline{\bar{\chi}}^{(3)}(\mathrm{z}): \vec{E}_{0, \mathrm{p}}^{k}(\mathrm{z}) \vec{E}_{0, \mathrm{p}}^{k}(\mathrm{z}) \vec{E}_{0, \mathrm{~s}}^{\prime *}(\mathrm{z}) \vec{E}_{0, \mathrm{c}}^{n^{*}}(\mathrm{z}) \mathrm{dz}
$$

When the waveguide consists of isotropic layers, the $\chi^{(3)}$ tensor consists of 21 nonzero elements of which only three are independent:

$$
\chi_{111}^{(3)}=\chi_{1221}^{(3)}+\chi_{1212}^{(3)}+\chi_{1122}^{(3)} \text {. }
$$

When the pump, Stokes, and CARS beams are all propagating in TE modes, only one component of the thirdorder susceptibility will contribute to the signal. Therefore, the overlap function can be simplified with Eq. 1 to

$$
F^{k l m}=\int_{-\infty}^{+\infty} \chi_{1111}^{(3)}(\mathrm{z}) f_{\mathrm{p}}^{k}(\mathrm{z}) f_{\mathrm{p}}^{k}(\mathrm{z}) f_{\mathrm{s}}^{\prime^{*}}(\mathrm{z}) f_{\mathrm{c}}^{m^{*}}(\mathrm{z}) \mathrm{dz}
$$

The overlap integral contains all the information about the waveguide structure, the excitation frequencies, and the nonlinear susceptibilities of the waveguide materials. The $\chi_{1111}^{(3)}$ element will be simply written as $\chi^{(3)}$. When this element of the nonlinear susceptibility is split into its nonresonant and resonant part, it can be written as:

$$
\chi^{(3)}=\chi_{\mathrm{NR}}^{(3)}+\chi_{\mathrm{R}}^{(3)} \text {. }
$$

By mixing a known resonant signal with an unknown nonresonant signal, one can determine the nonresonant part by curve fitting the measured heterodyne CARS spectrum. The resonant signal can be provided by a thin layer on top of the waveguide (overlayer). For such a waveguide structure, the overlap integral (Eq. 6) can be written as a sum of three integrals, all representing one layer (for the $\mathrm{TE}_{k l m}$ mode combination):

$$
\begin{aligned}
F^{k l m}= & \chi_{\mathrm{Nr}, \text { substratet }}^{(3)} g_{\text {substrate }}+\chi_{\mathrm{NR}, \mathrm{film}}^{(3)} g_{\text {film }} \\
& +\left\{\chi \chi_{\mathrm{NR}}^{(3)}+\chi_{\mathrm{R}}^{(3)}\right\}_{\text {overlayer }} g_{\text {overlayer }}
\end{aligned}
$$

with

$$
\begin{aligned}
g_{\text {substrate }} & =\int_{\text {substrate }}\left\{f_{\mathrm{p}}^{k}(\mathrm{z})\right\}^{2} f_{\mathrm{s}}^{\mu^{*}}(\mathrm{z}) f_{\mathrm{c}}^{m^{*}}(\mathrm{z}) \mathrm{dz} \\
g_{\text {film }} & =\int_{\text {film }}\left\{f_{\mathrm{p}}^{k}(\mathrm{z})\right\}^{2} f_{\mathrm{s}}^{\prime *}(\mathrm{z}) f_{\mathrm{c}}^{m^{*}}(\mathrm{z}) \mathrm{dz} \\
g_{\text {overlayer }} & =\int_{\text {overlayer }}\left\{f_{\mathrm{p}}^{k}(\mathrm{z})\right\}^{2} f_{\mathrm{s}}^{\prime^{*}}(\mathrm{z}) f_{\mathrm{c}}^{m^{*}}(\mathrm{z}) \mathrm{dz}
\end{aligned}
$$

The factors $g$ (substrate), $g($ film), and $g$ (overlayer) of Eq. 9 express the effective contributions of the different layers

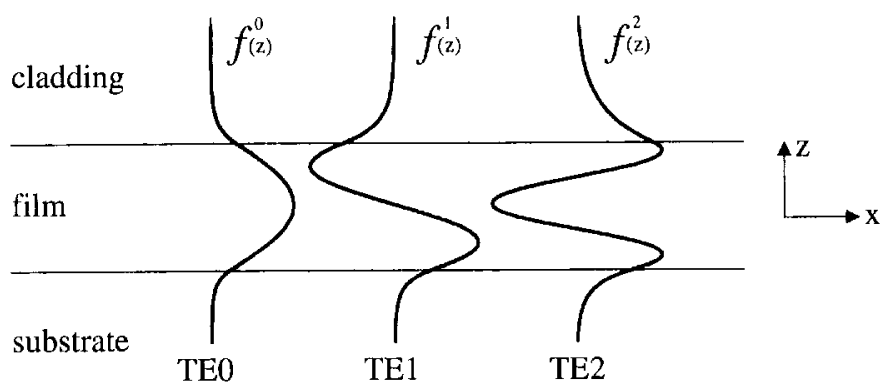

Fig. 1. A three-layered waveguide structure consisting of a cladding $(n=1)$, film $(n=1.73)$, and substrate $(n=1.46)$. The electrical field distribution functions $f^{k}(\mathrm{z})$ of the TEO, TE1, and TE2 modes are depicted.

to the overall CARS signal. It should be noted that we assume the $\chi^{(3)}$ of the cladding (most often air) to be zero as well as the $\chi_{R}^{(3)}$ of the film and substrate. The ratio between the resonant and the nonresonant contribution to the CARS signal can now be written as

$$
\begin{aligned}
& \frac{\chi_{R}^{(3)}}{\chi_{N R}^{(3)}} \\
& =\frac{\chi_{R}^{(3)}, \text { overlayer } g_{\text {overlayer }}}{\chi_{\mathrm{NR}, \text { overlayer }}^{(3)} g_{\text {overlayer }}+\chi_{N \mathrm{NR}, \text { substrate }}^{(3)} g_{\text {substrate }}+\chi_{\mathrm{NR}, \mathrm{film}}^{(3)} g_{\text {film }}} .
\end{aligned}
$$

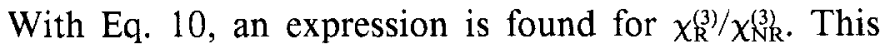
ratio determines the band shape of the resonant Raman bands in a WCARS spectrum. Measurement of these band shapes can in return give the ratio $\chi_{\mathrm{R}}^{(3) /} \chi_{\mathrm{Nk}}^{(3)}$ if the spectra are analyzed by curve fitting. The integrals $g$ (substrate), $g($ film), and $g$ (overlayer) can be calculated numerically. If the $\chi^{(3)}$ values of the thin overlayer and the substrate are known, the unknown $\chi_{N k}^{33}$ of the film layer can be determined.

In order to analyze the spectra, the nonresonant part is assumed to be independent of the frequency and the resonant part is written as a summation over all Raman active vibrations:

$$
\chi^{(3)}=\chi_{\mathrm{NR}}^{(3)}+\sum_{\mathrm{v}} \chi_{\mathrm{R}, \mathrm{v}}^{(3)} \Gamma_{\mathrm{v}} /\left[\left(\omega_{\mathrm{v}}-\omega_{\mathrm{p}}+\omega_{\mathrm{s}}\right)+i \Gamma_{\mathrm{v}}\right] .
$$

In this equation $\mathrm{v}$ enumerates the Raman vibrations with frequency $\omega_{\mathrm{v}}$ and with bandwidth $\Gamma_{\mathrm{v}}$. In general the CARS signal is proportional to the square modules of $\chi^{(3)}$ :

$$
P_{\text {CARS }} \sim\left|\chi^{(3)}\right|^{2} \text {. }
$$

The CARS spectra can be fitted with the use of Eq. 11 and 12 to obtain the amplitudes of $\chi_{k, v}^{(3)}, \chi_{N k}^{(3)}, \omega_{v}$, and bandwidth $\Gamma_{\mathrm{v}}$. Since for the outlined method only the ratio of one vibration is necessary to determine the unknown $\chi_{N R}^{(3)}$ of the film layer, the subscript $\mathrm{v}$ is dropped for the particular vibration and the ratio is written as $\chi_{\mathrm{R}}^{(3)} \chi_{\mathrm{NR}}^{(3)}$ as in Eq. 10.

\section{EXPERIMENTAL}

The waveguide was made on a silicon wafer which was thermally oxidized for $11 \mathrm{~h}$ at $1150^{\circ} \mathrm{C}$ to make a 1500 $\mathrm{nm}$-thick substrate of $\mathrm{SiO}_{2}$ (refractive index $n=1.461$ ). On top of this substrate a 480-nm-thick layer of SiON 


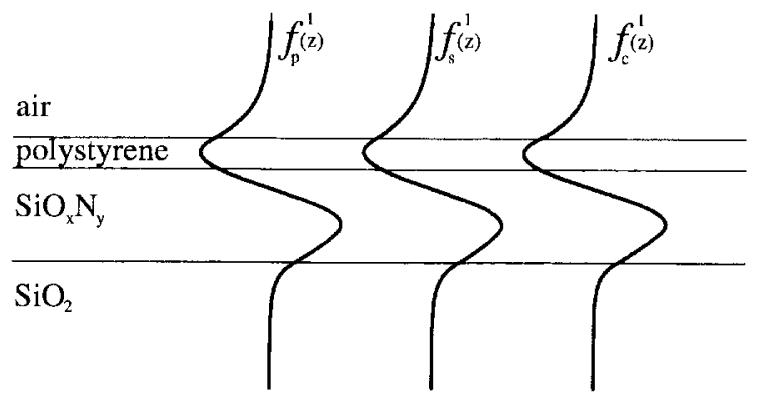

FIG. 2. The waveguide structure used in the WCARS experiments described in the text. The substrate consists of $\mathrm{SiO}_{2}$, the film of $\mathrm{SiO}_{x} \mathrm{~N}_{y}$, the overlayer of polystyrene, and the cladding of air. Only the situation with the overlayer thickness of $160 \mathrm{~nm}$ is shown. The electrical distribution functions of the applied modes are shown to illustrate the probing of the polystyrene vibrations with the evanescent field.

(refractive index $n=1.73$ ) was grown by the LPCVD (low-pressure chemical vapor deposition) technique. The thickness and refractive index of the $\mathrm{SiO}_{x} \mathrm{~N}_{y}$ layer were determined experimentally by fitting the measured coupling angles of the different guiding modes [TE as well as the transverse, or y-polarized, magnetic field (TM) modes] with the use of the thickness and refractive index as fit parameters. Polystyrene was chosen for the overlayer to provide the resonant signal (polystyrene gives a strong resonant signal at $1000 \mathrm{~cm}^{-1}$ ). In order to make thin overlayers, polystyrene was dissolved in toluene and spincoated on top of the SiON waveguide. The thickness of the polystyrene layer (refractive index $n=1.61$ ) was measured with a DEKTAK surface profiler. In Fig. 2 the waveguide configuration is shown for a polystyrene overlayer thickness of $160 \mathrm{~nm}$ together with the applied pump and Stokes modes (TE1) and the detected CARS mode (TE1).

The second harmonic of a Nd:YAG laser (Quanta-Ray DCR2, operated at $10 \mathrm{~Hz}$, pulse duration: $8 \mathrm{~ns}$ ), is used for the pump beam at $532 \mathrm{~nm}$. Part of the second harmonic is used to pump a Quanta-Ray dye laser (Rhodamine $6 \mathrm{G}$ ), which provides the tuneable Stokes beam. Polarization directions of the beams were adjusted by Glan-Taylor polarizers. An SF6 prism was used to couple the pump and Stokes beams into the TE1 mode of the waveguide. The incoupling efficiency was approximately $5 \%$ and the intensities of both pump and Stokes beams were on the order of $1 \mathrm{MW} / \mathrm{cm}^{2}$ inside the waveguide. Pump and Stokes beams were applied under a small angle $\left(\sim 1^{\circ}\right)$ to ensure phase matching. The widths of the beams inside the waveguide were $1-2 \mathrm{~mm}$. Great care was taken not to overlap the pump and Stokes beams inside the SF6 prism because this can give rise to an additional nonresonant signal originating from the prism. The generated

TABLE I. The ratio $\chi_{\mathrm{R}}^{(3)} / \chi_{\mathrm{N}}^{(3)}$ as extracted from the measured WCARS spectra for the different polystyrene overlayer thicknesses.

\begin{tabular}{cc}
\hline $\begin{array}{c}\text { Thickness polystyrene } \\
\text { overlayer }(\mathrm{nm})\end{array}$ & $\chi_{\mathrm{R}}^{(3) / \chi_{\mathrm{NR}}^{(3)}}$ \\
\hline $470 \pm 10$ & $2.00 \pm 0.2$ \\
$340 \pm 10$ & $1.63 \pm 0.16$ \\
$160 \pm 10$ & $0.83 \pm 0.08$ \\
$80 \pm 10$ & $0.26 \pm 0.07$ \\
\hline
\end{tabular}

TABLE II. The calculated layer contributions $g$ (overlayer), $g$ (film), and $g$ (substrate) to the CARS signal for the differemt polystyrene overlayer thicknesses. The layer contributions are normalized on the sum of $g$ (overlayer), $g($ film), and $g($ substrate) and given in percentages.

\begin{tabular}{cccc}
\hline $\begin{array}{c}\text { Thickness } \\
\text { polysty- } \\
\text { rene over- } \\
\text { layer (nm) }\end{array}$ & $\begin{array}{c}g(\text { overlayer) } \\
(\%)\end{array}$ & $\begin{array}{c}g(\text { film }) \\
(\%)\end{array}$ & $\begin{array}{c}g(\text { substrate) } \\
(\%)\end{array}$ \\
\hline 80 & 5.8 & 87.4 & 06.7 \\
160 & 14.1 & 80.8 & 05.1 \\
340 & 31.7 & 64.9 & 03.4 \\
470 & 42.4 & 54.8 & 02.7 \\
\hline
\end{tabular}

CARS signal was coupled out of the waveguide with a second SF6 prism (approximately $1 \mathrm{~cm}$ separated from the incouple prism), analyzed by a Glan-Taylor polarizer and collected by a spherical mirror. By selecting the proper outcoupling angle from the SF6 prism, one ensures that only the CARS signal generated in the TE1 mode is detected. The signal is then focused onto the entrance slit of a double monochromator (UV-Vis $200 \mathrm{~mm}$ JobinYvon) and detected by a photomultiplier (RCA 9973B). The computer and electronics are interfaced by an IEEE bus. The CARS signals are digitized in an 8-channel 12bit ADC and stored on floppy disk for later analysis. Spectra are measured in a scanning mode with the pump frequency fixed and Stokes frequency tuneable and, in this case, were recorded over an interval 975 to 1050 $\mathrm{cm}^{-1}$ in 75 points with 45 pulses averaged per point. All spectra shown were corrected for laser intensity fluctuations and smoothed with a 7-point smoothing procedure. Four spectra were measured with polystyrene thicknesses of $470,340,160$, and $80 \mathrm{~nm}$. In the spectral region of interest $\left(975-1050 \mathrm{~cm}^{-1}\right)$, no resonant signals of the substrate and film layer were detected. This result validates the assumptions made in the theoretical section.

\section{ANALYSIS}

All spectra were fitted with a nonlinear least-squares fit procedure. In Fig. 3 the spectra are shown together with the fitted curves. The ratios $\chi_{R}^{(3)} / \chi_{N R}^{(3)}$ obtained from the fitting are shown in Table I. In order to extract $\chi \chi_{N k}^{(3)}$

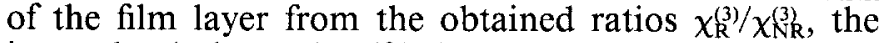
integrals $g$ (substrate), $g$ (film), and $g$ (overlayer) were calculated numerically for the four different polystyrene overlayer thicknesses. The calculated integrals [normalized on the sum of $g$ (film), $g$ (substrate), and $g$ (polystyrene)] are shown in Table II. As expected, the effective contribution of the thin polystyrene overlayer increases for increasing overlayer thickness. With the use of Eq. 10 together with the calculated functions $g$ (substrate), $g($ film), and $g$ (overlayer), the experimental results can be fitted with $\chi_{\mathrm{Nk}}^{(3)}$ of the film $\left(\mathrm{SiO}_{x} \mathrm{~N}_{y}\right)$ as the fitting parameter, since all other third-order susceptibilities are known [substrate: $\chi_{\mathrm{NR}}^{(3)}\left(\mathrm{SiO}_{2}\right)=0.02310^{-12}$ electronic units (esu); ${ }^{1}$ overlayer: $\chi_{N k}^{(3)}$ (polystyrene) $=0.3 \quad 10^{-12}$ esu; and $\chi_{\mathrm{R}}^{(3)}$ (polystyrene) $\left.=1.210^{-12} \mathrm{esu}^{8}\right]$. The result of this fit is shown in Fig. 4. The obtained value for the $\chi_{\mathrm{Nk}}^{(3)}$ of $\mathrm{SiO}_{x} \mathrm{~N}_{y}$ was found to be $0.22 \pm 0.0310^{-12}$ esu. In Fig. 4, which appears on the final page, two more curves are shown which were calculated for two different values of $\chi_{\mathrm{NR}}^{(3)}(0.1$ and $\left.0.410^{-12} \mathrm{esu}\right)$ to show the sensitivity of the fitted curves to the fitting parameter. 

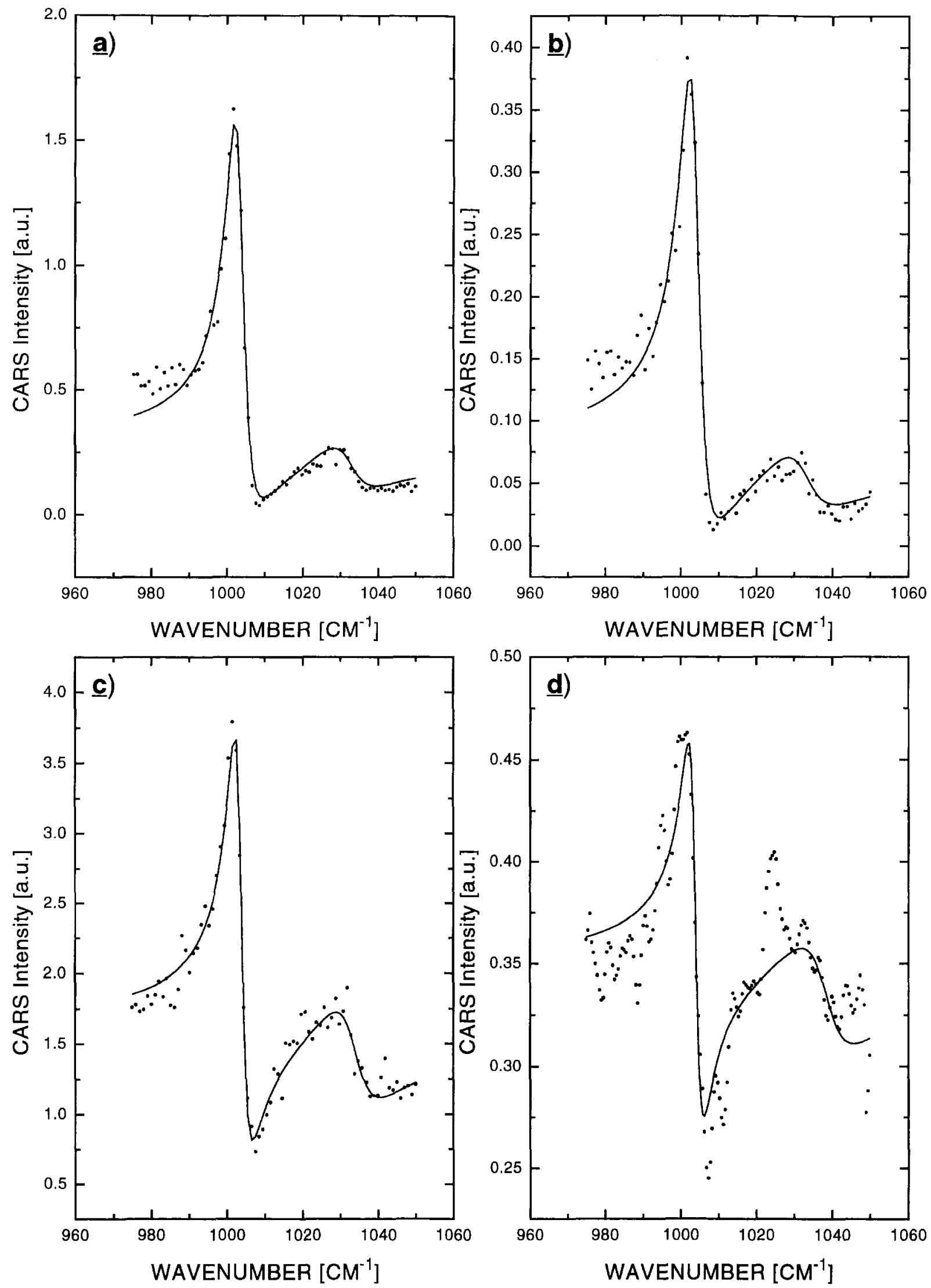

Frg. 3. Measured (dotted) and fitted (solid) WCARS spectra of the described waveguide for different polystyrene layer thicknesses: (a) 470 nm; (b) $340 \mathrm{~nm}$; (c) $160 \mathrm{~nm}$; (d) $80 \mathrm{~nm}$. 


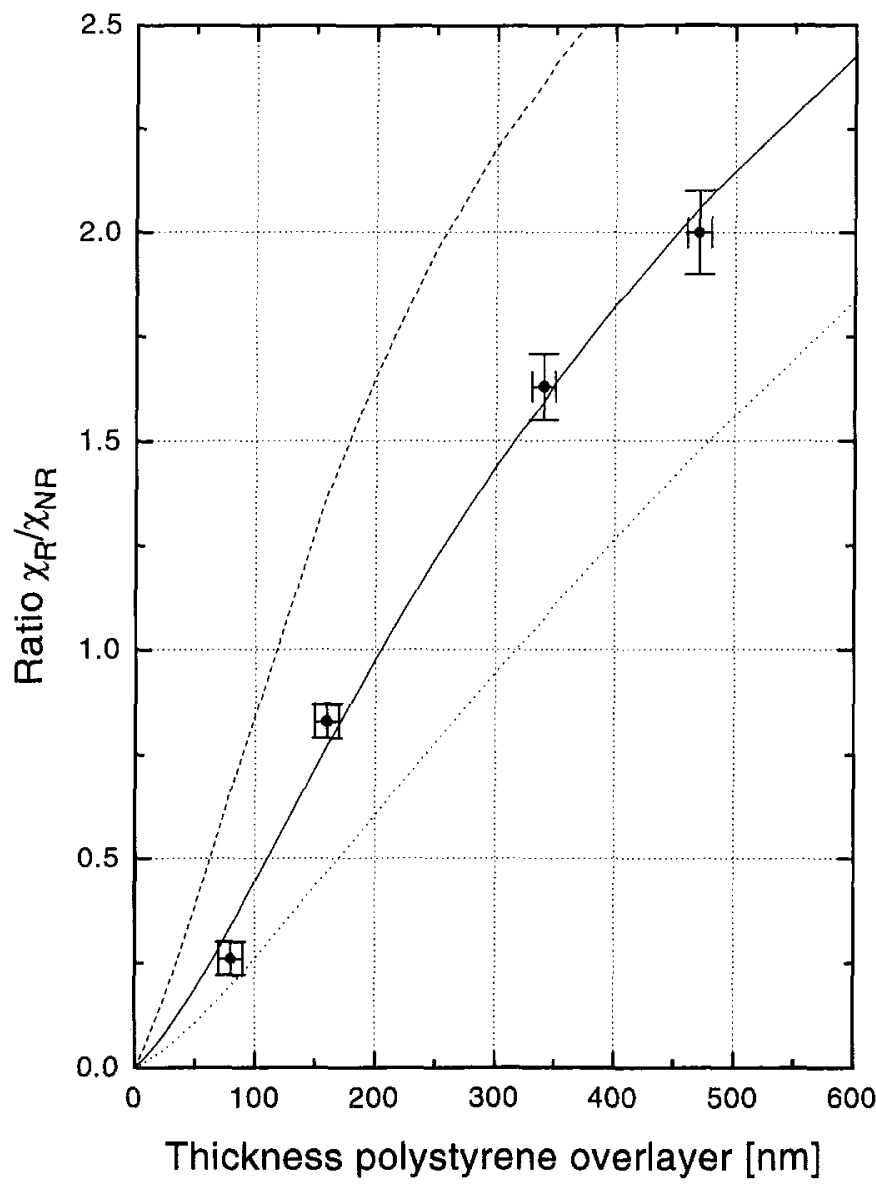

Fig. 4. Measured (dots) and calculated (solid, dotted, and dashed curves) ratios $\chi_{k}^{(3)} / \chi_{N k}^{(3)}$. The $\chi_{\mathrm{Nk}}^{(3)}$ values of $\mathrm{SiO}_{x} \mathrm{~N}_{y}$ used to calculate the ratios are: $0.2210^{-12} \mathrm{esu}$ (solid); $0.110^{-12}$ esu (dashed); and $0.410^{-12}$ esu (dotted).

\section{CONCLUSION}

A method has been outlined to measure the nonresonant third-order polarizability of waveguide layers. This method is based on determining the ratio between the resonant and nonresonant signal of WCARS spectra. The resonant signal is in this case provided by a thin overlayer on top of the waveguide. It was shown that the measurement of the ratio dependence on the overlayer thickness is in close agreement with theory. Thin layers of polystyrene on top of a $\mathrm{SiO}_{x} \mathrm{~N}_{y}$ waveguide structure were measured for several polystyrene layer thicknesses down to $80 \mathrm{~nm}$. The $\chi_{\mathrm{Nk}}^{(3)}$ of $\mathrm{SiO}_{x} \mathrm{~N}_{y}$ was obtained from these measurements and found to be $0.22 \pm 0.0310^{-12}$ esu.

\section{ACKNOWLEDGMENT}

We wish to acknowledge the support provided to one of us (J. S. Kanger) by the S.T.W., Technology Foundation, Grant TTN11.2511.

1. G. I. Stegeman, R. Fortenberry, C. Karaguleff, R. Moshrefzadeh, W. M. Hetherington III, N. E. van Wyck, and J. E. Sipe, Opt. Comm. 82, 295 (1983).

2. W. M. Hetherington III, N. E. van Wyck, E. W. Koenig, G. I. Stegeman, and R. M. Fortenberry, Opt. Lett. 9, 88 (1984).

3. D. Narayana Rao, R. Burzynski, and P. N. Prasad, Opt. Comm. 82, 357 (1991).

4. W. P. de Boeij, J. S. Kanger, G. W. Lucassen, C. Otto, and J. Greve, Appl. Spectrosc. 47, 723 (1993).

5. Z. Z. Ho, W. M. K. P. Wijekoon, E. W. Koenig, and W. M. Hetherington III, J. Chem. Phys. 92, 757 (1987).

6. W. M. Hetherington III, Z. Z. Ho, E. W. Koenig, G. I Stegeman, and R. M. Fortenberry, Chem. Phys. Lett. 128, 150 (1986).

7. J. E. Sipe and G. I. Stegeman, J. Opt. Soc. Am. 69, 1676 (1979).

8. D. D. Maker and R. Terhune, Phys. Rev. A137, 801 (1964). 